\title{
Lymph Node Part
}

National Cancer Institute

\section{Source}

National Cancer Institute. Lymph Node Part. NCI Thesaurus. Code C38466.

Any component of the bean-shaped organ that is part of the lymphatic system found

throug hout the body which is composed predominantly of lymphocytes and is involved in immune protection. 